Canadian Oncology

Nursing Journal

Revue canadienne

de soins infirmiers

en oncologie

Volume 28, Issue 3 • Summer 2018

elSSN: 2368-8076 


\title{
Engaging patients with radiation related skin discomfort in self-care
}

\author{
by Crystele Montpetit and Savitri Singh-Carlson
}

\section{ABSTRACT}

Background: Patients receiving radiation to the breast can suffer painful, moist desquamation of the axilla and inframammary fold. Intensity of skin reactions will vary with each individual and be dependent on the total dose of radiation, treatment volume, daily fraction size, energy and type of radiation, as well as other personal factors (Gosselin, 2010). Treatment-related skin reactions make it difficult for women to wear clothing and undergarments comfortably and to perform daily activities. InterDry AG is a textile dressing with an antimicrobial complex that wicks away excess moisture and reduces friction, itching, and burning. Despite showing highly beneficial properties, there is no current published evidence indicating the utility of InterDry Ag for radiation skin reactions.

Purpose: This project evaluated the potential utility of InterDry Ag dressing to relieve discomfort of skin reactions for breast cancer patients undergoing radiation treatment.

Methods: A descriptive design was employed to better understand the effectiveness of InterDry Ag dressing in reducing side effects of radiation treatment of the breast for women. Twenty eligible participants consented to participate. A radiation oncology nurse evaluated its effect every five days, utilizing a skin assessment questionnaire developed by the nursing team from clinical observations.

Results: A personalized skin care regimen was developed with each patient's feedback. A trusting relationship was developed between the nurse and patient by building rapport that indicated nursing care support. Some women began sharing their emotional stressors with nurses, as well as the physical pain they were experiencing due to the skin reactions. This observation resulted in provision of additional resources for women and engaging them in self-care using a patient-centred approach.

\section{ABOUT THE AUTHORS}

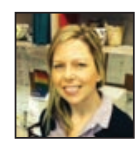

Crystele Montpetit, RN, BScN, Radiation and Systemic Oncology Nurse, British Columbia Cancer Agency, Fraser Valley Cancer Centre

Phone: 604-930-2098

Email: crystele.montpetit@bccancer.bc.ca

Savitri Singh-Carlson, PhD, APHN-BC, FAAN, Vice Dean Faculty Affairs and Diversity, University of Texas Health Sciences Center San Antonio, School of Nursing, San Antonio, USA, 78248

Phone: 2105675883

Email: singhcarlson@uthscsa.edu

Corresponding author: Crystele Montpetit: crystele.montpetit@bccancer.bc.ca DOI: $10.5737 / 23688076283191200$

\section{INTRODUCTION}

A ccording to recent Canadian statistics, breast cancer (BC) $\mathrm{A}_{\text {is }}$ the most common cancer among Canadian women, with an estimated 25,700 women being diagnosed with BC in 2016 (Canadian Cancer Society, 2016). Literature indicates that most women generally experience negative psychological, physical, or emotional side effects, that impact their personal health and well-being after BC treatment (Budischewski, 2008; Smith, Singh-Carlson, Downie, Payeur, \& Wai, 2011; Tanay, 2013). Discomfort experienced by women during breast radiation treatment affects their quality of life and hinders their ability to perform activities of daily living.

The most common side effect of radiation therapy treatment is radiation dermatitis, which can cause painful complications for BC patients (Tanay, 2013). Radiation treatment and subsequent inflammatory responses are pathophysiological reactions that occur after radiation of the skin and, generally, at the entrance and exit site of irradiation (BCCA Symptom Management Guidelines, 2013). Patients receiving radiation to the breast may also suffer from progressive skin reaction, resulting in painful moist desquamation of the axilla and the inframammary fold, making it difficult to wear clothing and undergarments and to perform daily activities. Radiation skin reactions vary from mild to severe, and are graded according to the NCI CTCAE (version 4.03) for Dermatitis Radiation. Furthermore, when these painful open areas are left untreated, complications may occur, resulting in secondary bacterial or fungal infections (Kennedy-Evans, Smith, Viggiano, \& Henn, 2007). Patients also experience varying levels of associated emotional distress together with significant physical discomfort (Schnur, Ouellette, Dilorenzo, Green, \& Montgomery, 2011). Feelings of pain, burning, itchiness, and pins and needles with heaviness have been described by some BC patients (Paterson, et.al., 2012). These symptoms often increase in severity for up to two weeks following their last radiation treatment.

Radiation treatment skin reactions are treated according to severity of toxicity with goals of symptom management and infection prevention. Symptom Management Guidelines are one of the resources frequently used by radiation oncology nurses (RON) at the British Columbia Cancer Agency (BCCA), (BCCA Symptom Management Guidelines, 2013). Standardized treatment of moderate to severe skin reactions has typically been saline compresses followed by a moisturizer or prescription creams such as flamazine and/or hydrocortisone cream. RONs at this clinical project site have observed that many patients find it difficult and messy to apply the prescription creams and they do not always experience relief from the discomfort even after its application.

In general, reactions to radiation treatment become evident one to four weeks after beginning treatment, and may 
persist for several weeks post treatment (BCCA Symptom Management Guidelines: Radiation Dermatitis, 2013). Experts report that moisture-wicking dressings with silver $(\mathrm{Ag})$ have the ability to translocate excess moisture from the skin fold to keep skin dry with the silver-impregnated formulation providing effective antimicrobial action for five days (Sibbald, 2013). This strategy permits the soft knitted textile to provide a friction-reducing surface that reduces risks of skin tears. Furthermore, the textile is used to treat signs and symptoms of intertriginous dermatitis, such as maceration, denudement, inflammation, pruritus, erythema, and satellite lesions to prevent further complications (Sibbald, 2013).

There is a lack of evidence in the literature regarding clinical utilization of InterDry AG for radiation skin reactions, despite its medicinal properties that could prove highly beneficial for this patient population (Kumar, Juresic, Barton, \& Shafiq 2010). Yet, healthcare cost for clinics utilizing InterDry AG dressing is approximately half in comparison to other prescription treatments. Currently, skin reactions are viewed as an unavoidable part of treatment. Therefore, nursing management in follow-up nursing clinics is directed toward the palliation of skin reaction symptoms (Porock, 1999). Other reports have identified five management trials that examined a range of products (corticosteroid creams, sucralfate cream, hydrocolloid dressings, and a moisture vapor permeable dressing) (Paterson, 2012). Based on these results, no agent was found that significantly reduced the impact of acute radiation-induced skin reactions.

Given the paucity of literature on the efficacy of InterDry Ag, a quality improvement project explored the benefits of using InterDry Ag dressing moisture wicking fabric, in assessment of healing and relief from discomfort of radiation skin reactions of the breast. The purpose of this project was to evaluate BC patients' perceptions of the efficacy of utilizing InterDry Ag dressing in relieving their discomfort from skin reactions due to radiation treatment. The project provided valuable information for quality improvement of cancer care provided for BC patients with skin reactions and its complexities arising from radiation treatment.

\section{FOLLOW-UP NURSING CLINIC}

The follow-up nursing clinic at the Fraser Valley Centre (FVC) of BCCA addresses secondary symptoms arising from radiation or chemotherapy treatments such as skin reactions, patient distress due to pain, nausea, and other pathophysiological dysfunctions. At the beginning of treatment, patients are provided with written resources on the side effects of radiation therapy. Patients are advised to call the nursing telephone line at the clinic, as necessary. Patients may be seen and assessed by an RON and/or their radiation oncologist (RO) in one of four outpatient clinics or at the patient review clinic in the radiation department.

Current standard of care following radiation treatment at this clinic for BC patients is an appointment with their RO, approximately four to eight weeks after the last day of treatment. Nursing skin assessment is a prerequisite in order to gain an understanding of the patient's unique experience and to provide appropriate care that includes fostering patient selfcare management. In order to get the best results for this project, a nursing assessment was scheduled at least two weeks following patients' last radiation treatment. Currently, standard of care at the clinic does not include a follow-up assessment with the RON; patients are assessed by the RON on an as needed basis.

Radiation therapists may refer patients to the RON following the scheduled treatment in order to assess for individual skin reactions. The assessment is generally completed in the patient review area in the radiation clinic. Patient education is a large component of this session, where patients are given written and/or verbal information on how to manage physical side effects of skin reactions. Patient education includes addressing possible emotional distress and malnutrition that could affect the wound healing process. During the assessment, the RON provides information on the daily care of the irradiated skin and the use of a daily saline compress and its application. The importance of clearly written discharge instructions and confirmation of patient's comprehension of self-management plans, if moist desquamation should occur, has been acknowledged (Laffin et al., 2015).

\section{METHODOLOGY}

A descriptive design was employed for this evaluative project to gain insight into BC patients' experiences of using the InterDry Ag dressing, to relieve symptoms of skins reactions from active radiation treatment. A feedback loop evaluation methodology that permits evidence to move into practice to improve quality of care was a fit for this project (Gotz \& SinghCarlson, 2014).

The nursing team informed the development of questionnaires based on their clinical observations of BC complex dermatitis due to radiation therapy. These clinical observations were used to develop open-ended questions for data collection. Both structured and open-ended items were included in the questionnaire used by the RON to collect participants' qualitative responses about their personal experiences using the dressing (see Appendix A \& B). The questionnaire (see Appendix C) used Likert response scales to explore potential effectiveness of InterDry Ag dressing in providing patient comfort.

Following appropriate ethics approval, participants were recruited based on their eligibility and willingness to participate. Eligibility criteria were: a) radiation treatment for breast cancer at the FVC; b) no known allergy to silver; c) a skin reaction from radiation treatment; and d) an ability to speak and read English fluently. An RON recruited potential participants at the beginning of their radiation treatment by providing written introduction letters that described the evaluative project. After written consent was obtained and instructions were provided, patients were assessed on a weekly basis for radiation skin reactions. The InterDry Ag dressing was only applied for skin reactions with moderate erythema, pruritus or burning pain noted by the patient. Patients continued to utilize the 
dressing for two weeks following the end of treatment or the development of moist desquamation.

\section{Procedure}

A weekly assessment for moderate erythema of the breast was completed for all participants while they were on active radiation treatment. Information on saline compresses, moisturizers and the application of InterDry Ag dressing was provided during each weekly assessment.

The InterDry Ag dressing was provided when moderate erythema and discomfort were noted. The dressing was provided as a treatment to potentially alleviate symptoms of burning, itching, and general discomfort. If a moderate skin reaction was evident on the breast without any open areas, the dressing was placed over the entire breast. Patients were advised to continue with regular saline compresses, as needed, and to utilize regular use of moisturizers. The moisturizers were to be absorbed and the skin area dried prior to the application of InterDry Ag dressing.

Evaluation of the use of InterDry Ag was done through a weekly standard skin assessment using the National Cancer Institute: Common Terminology Criteria for Adverse Events (NCI CTCAE) (version 4.03) Dermatitis Radiation tool and a participant questionnaire was completed by an RON (Appendix A). The participants' level of comfort was also assessed at this time. If the patient developed moist desquamation at any point during treatment, flamazine cream was prescribed by the RO and applied to all open areas. The InterDry Ag dressing was not applied to any open areas. Hydrocortisone cream has typically been standard of care for treatment of pruritus and prescribed at the discretion of the patients' RO. Three patients chose to apply the hydrocortisone cream prior to the use of InterDry Ag dressing.

Skin assessment included the three most common sites affected during breast radiation treatment: the axilla, inframammary fold, and the chest wall. Erythema, pain, and pruritus were assessed and documented as "none, mild, moderate or severe". Information documented was based on clinical observation by the RON and patients' feedback. Each participant received between two and five skin assessments. This variation was dependent on the start date of InterDry Ag dressing utilization and the total number of radiation treatments received. Participants were given the opportunity to discuss any concerns about their skin care at this time. Each appointment time varied between 15 and 30 minutes, based on participants' individual needs.

The InterDry AG dressing was replaced every five days during assessment appointments. During the last radiation treatment appointments, participants were provided with two additional pieces of InterDry Ag dressings, with any additional supplies needed to secure the dressing. This enabled patients to care for their skin independently until the next follow-up visit.

The follow-up clinic RON contacted participants by telephone one week following their last day of radiation treatment to gather information about patients' experiences using a standardized template (Appendix B). Eleven questions were directed to the effectiveness of InterDry Ag and its ability to provide comfort for patients. Participants were given the opportunity to discuss any questions or concerns related to the dressing and skin integrity at this time. The RON completed a skin assessment, as well as a questionnaire two weeks post radiation treatment (Appendix C). Participants were given an opportunity to document any additional comments or suggestions, based on their personal experiences of utilizing the InterDry Ag dressing.

\section{Data analysis}

Descriptive statistics were utilized to analyze responses from the questionnaires. The data included an assessment conducted by the RON for erythema, pain and pruritus on the axilla (AX), inframammary fold (IF), and chest wall (CW). The data was entered into Excel 2007, with each of these side effects categorized as none $=0$, mild $=1$, moderate $=2$ and severe $=3$ at each assessment point. Patient responses (Appendix C) were given a numerical value with the Likert scale ranging from one to six: Strongly Agree $=1 ;$ Agree $=2$; Undecided = 3; Disagree $=4$; Strongly Agree $=5$; and Not Applicable $=6$. Each response was counted as a value of one. The questionnaire data were checked and entered by the author (CM). Data entry was checked for accuracy by a second individual before statistical analysis was completed using Excel 2007. Participants' qualitative responses were summarized and illustrated with quotes to support the data. Content analysis helped to categorize participants' qualitative responses (Stemler, 2001).

\section{RESULTS}

Twenty eligible participants initially agreed to take part in the evaluative project. However, one withdrew due to personal reasons and another did not attend the follow-up appointment. Eighteen participants completed the first and second skin assessment and 18 completed the telephone follow-up questionnaire, as well as the questionnaire two weeks following their last radiation treatment.

\section{Demographic Data}

The median age for participants was 42 years (range 36-74). Seventeen (91\%) of the participants were non-smokers. Participant's breast size varied from small (similar to an A cup size) to large (similar to an E cup size) and skin complexion varied from light to dark. Women receiving treatment for both chemotherapy and radiation treatment were included. Nine $(15 \%)$ were large-breasted women, requiring additional support such as a breast shell to stabilize the breast during radiation treatment. Additionally, 20\% (N=3) of participants had undergone the initial stage of reconstructive surgery prior to radiation treatment. Participants had diverse treatment plans, receiving a varied number of treatments (range 15-25) with varying doses per fraction. The median number of fractions received was 25 with a total Grey received of 5000cGy and a dose per fraction of 200cGY.

\section{Assessment Results}

Figure 1 illustrates the comparison between the initial two skin assessments completed by the RON. The first skin assessment examined participants' skin prior to use of InterDry Ag 


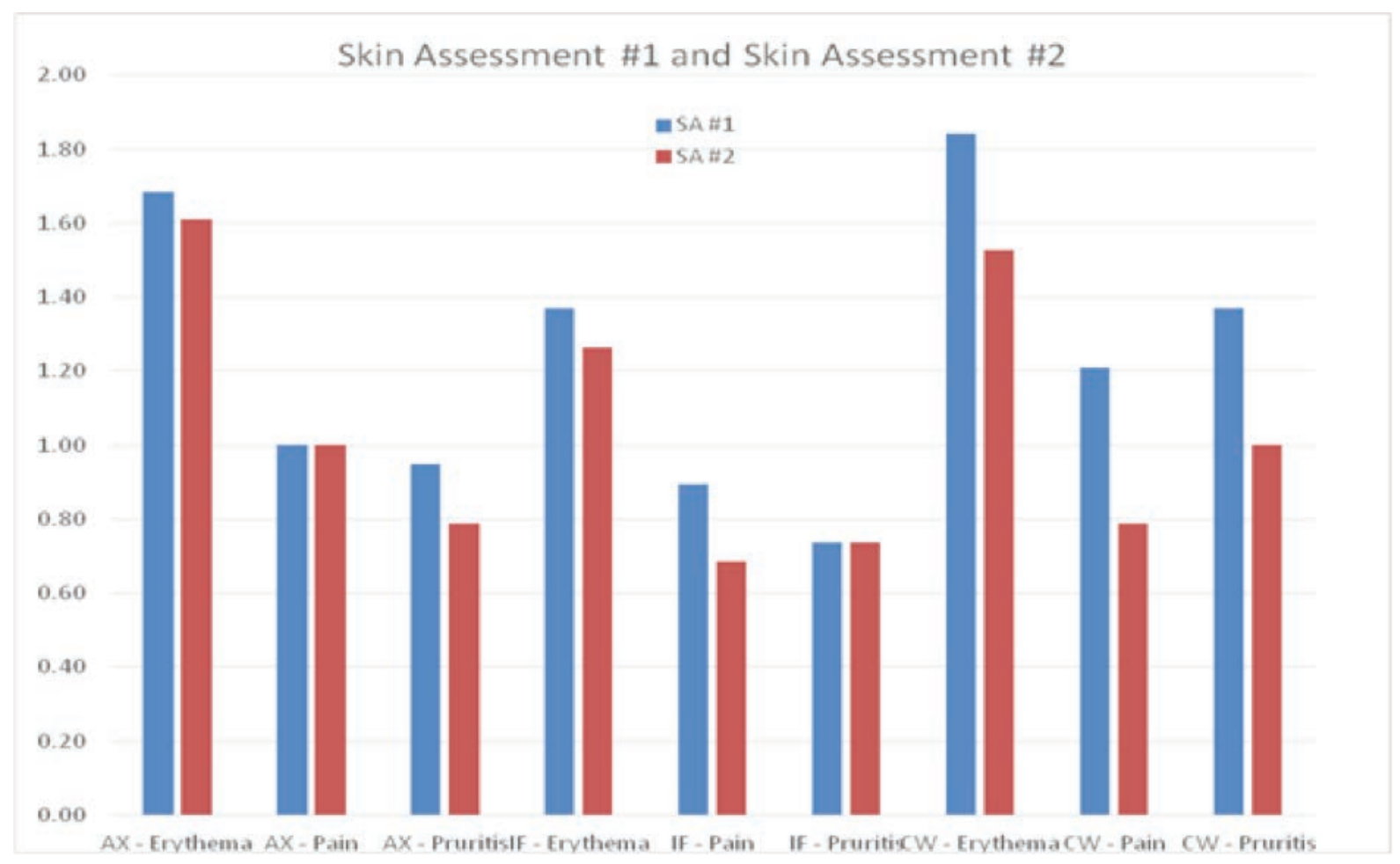

Figure 1: Skin assessments at baseline (time 1) and time $2(\mathrm{~N}=18)$

dressing and the second was performed five days following application of the dressing. Participants described a decrease in pain to the chest wall and inframammary fold following the initiation of InterDry Ag. A decrease in discomfort within the same day of application was reported by some participants along with a decrease in pruritus to the chest wall and axilla.

Each participant was also asked a series of 10 questions during each skin assessment (Appendix A). Five days following the initiation of the InterDry Ag dressing, participants were asked if the dressing relieved any discomfort. Eighteen (94.7\%) answered yes and $84.2 \%(\mathrm{~N}=16)$ stated their skin felt better after the dressing. When asked if this dressing relieved their pain, $63.2 \%(\mathrm{~N}=12)$ answered yes and $36.8 \%(\mathrm{~N}=7)$ were unsure. Several participants (26.3\%) (N=5), described the dressing as difficult to keep in place using either the method provided or a self-improvised approach. In contrast, 63.2\% $(\mathrm{N}=11)$ did not have any difficulty and $10.5 \%(\mathrm{~N}=2)$ had difficulty only sometimes.

Each participant received a follow-up telephone call from a RON one week after the last radiation treatment (Appendix B) and was asked a series of 10 questions (see Table 1). Eighteen participants completed the telephone follow-up questionnaire. Of these 18 participants, 94\% ( $\mathrm{N}=17$ ) were using the InterDry AG dressing one week following their last radiation treatment because of moist or dry desquamation of the skin. Seventeen (94\%) of the participants found this dressing was easy to use with $100 \%$ reporting that their skin felt better with the dressing. Sixteen (88\%) felt the dressing had helped the healing with $100 \%$ of participants expressing that they found the dressing to be comfortable.
Table 2 details the InterDry Ag dressing and its effect on decreasing pain, itching and burning experiences of the skin reaction. The questionnaire was completed at the two-week follow-up appointment with the nurse in the outpatient clinic at the FVC. Flamazine was not required during treatment with the exception of two participants. These two participants developed severe skin reactions requiring flamazine application for moist desquamation. Flamazine and hydrocortisone are topical medicated creams that have typically been prescribed by patients' RO to relieve severe discomfort and to prevent infection. This was an exception for participants with severe dermatitis and moist desquamation. Participants experienced varying levels of distress and radiation skin toxicity. Three of the participants chose to use hydrocortisone cream in addition to the application of the InterDry Ag dressing. These participants continued to experience pruritus of the skin while using the prescribed hydrocortisone cream and felt that pruritus may worsen if it was discontinued. They were advised to apply the InterDry Ag dressing after the hydrocortisone cream was completely absorbed into the skin. Some participants found relief of pruritus was evident with the additional use of the dressing. Participants expressed concern that the pruritus experience may worsen if the cream was discontinued.

\section{Participants' Personal Experiences}

A personal skin care regimen was developed with each participant at the beginning of their treatment. This included a schedule for the use of saline compresses and moisturizers. Appropriate use of the InterDry Ag dressing was also reviewed. Participants were able to ask any questions or voice any concerns with the RON every five days during their follow-up 
Table 1: Telephone Questionnaire Results $(\mathrm{N}=18)$

\begin{tabular}{|c|c|c|c|c|c|}
\hline Telephone Questionnaire & Yes & No & Improved & Worsened & Same \\
\hline $\begin{array}{l}\text { 1. Are you presently using the InterDry Ag? (If no, proceed to } \\
\text { question } \# 10 \text { ). }\end{array}$ & $94.1 \%$ & $5.9 \%$ & $0.0 \%$ & $0.0 \%$ & $0.0 \%$ \\
\hline 2. Do you find the dressing easy to use? & $93.8 \%$ & $6.3 \%$ & $0.0 \%$ & $0.0 \%$ & $0.0 \%$ \\
\hline $\begin{array}{l}\text { 3. Has the redness of your skin worsened or improved since your } \\
\text { last treatment date? }\end{array}$ & $0.0 \%$ & $0.0 \%$ & $43.8 \%$ & $43.8 \%$ & $12.5 \%$ \\
\hline $\begin{array}{l}\text { 4. Has your skin opened since your last treatment? ie: Are there } \\
\text { any wet patches? }\end{array}$ & $31.3 \%$ & $68.8 \%$ & $0.0 \%$ & $0.0 \%$ & $0.0 \%$ \\
\hline 5. Have you changed the dressing more than every five days? & $12.5 \%$ & $87.5 \%$ & $0.0 \%$ & $0.0 \%$ & $0.0 \%$ \\
\hline 6. Do you have any pain? & $86.7 \%$ & $13.3 \%$ & $0.0 \%$ & $0.0 \%$ & $0.0 \%$ \\
\hline 9. Does your skin feel better with the dressing on? & $100.0 \%$ & $0.0 \%$ & $0.0 \%$ & $0.0 \%$ & $0.0 \%$ \\
\hline $\begin{array}{l}\text { 10. Why did you stop using the dressing? (only applicable if not } \\
\text { using the dressing). }\end{array}$ & $0.0 \%$ & $0.0 \%$ & $0.0 \%$ & $0.0 \%$ & $0.0 \%$ \\
\hline 11. Are you using any other dressings or creams? & $93.8 \%$ & $6.3 \%$ & $0.0 \%$ & $0.0 \%$ & $0.0 \%$ \\
\hline
\end{tabular}

Table 2: Patient Survey Results (N=18)

\begin{tabular}{|c|c|c|c|c|c|c|}
\hline How strongly do you agree with this statement? & $\begin{array}{l}\text { Strongly } \\
\text { Agree }\end{array}$ & Agree & Undecided & Disagree & $\begin{array}{l}\text { Strongly } \\
\text { Disagree }\end{array}$ & N/A \\
\hline 1. InterDry AG is easy to use. & $39 \%$ & $61 \%$ & 0 & 0 & 0 & 0 \\
\hline 2. InterDry AG provides relief of pain. & $50 \%$ & $40 \%$ & 0 & $5 \%$ & 0 & $5 \%$ \\
\hline 3. InterDry AG provides relief of itch. & $50 \%$ & $33 \%$ & $6 \%$ & $11 \%$ & 0 & 0 \\
\hline 5. My skin feels better with the dressing on. & $67 \%$ & $28 \%$ & $5 \%$ & 0 & 0 & 0 \\
\hline 6. I feel this dressing has helped my skin to heal. & $61 \%$ & $17 \%$ & $22 \%$ & 0 & 0 & 0 \\
\hline $\begin{array}{l}\text { 7. Dressing has provided relief from any burning } \\
\text { sensations of skin. }\end{array}$ & $56 \%$ & $22 \%$ & $11 \%$ & 0 & 0 & $11 \%$ \\
\hline $\begin{array}{l}\text { 9. I feel more comfortable with the dressing in place } \\
\text { than without the dressing. }\end{array}$ & $72 \%$ & $17 \%$ & $11 \%$ & 0 & 0 & 0 \\
\hline 10. I would recommend this dressing to others. & $78 \%$ & $17 \%$ & $5 \%$ & 0 & 0 & 0 \\
\hline
\end{tabular}

visit. Patients indicated feeling decreased levels of anxiety and appreciation of care with the additional level of contact with the RON. Although participants reported decreased levels of anxiety, an anxiety scale is not provided at this time as part of standard care at this clinical site.

Participants' personal experiences also included benefits and challenges in using the InterDry Ag dressing for skin reaction. Most participants voiced their appreciation for the care given and the positive effects of this dressing. Some participants expressed the need to wear the dressing in order to prevent some of the painful side effects of radiation therapy. However, it was quite challenging to keep the dressing in place. Modifications had to be made to undergarments to keep the dressing in place with replacements every five days. Personal benefits/challenges with verbatim quotes in using the dressing are presented in Table 3. 


\section{Table 3: Participants' Verbatim Quotes}

\section{Patient Perceived Personal Benefits and Challenges}

Benefits

- When using the dressing I did not need pain medications. Without the dressing, I'm very uncomfortable. Works within minutes. Don't know what I would have done without it. Wish I would have started earlier.

- Satisfied with the InterDry Ag and I would recommend it to others.

- On the way home, I noticed I wasn't bothered by the itching and burning symptoms anymore. The annoyance was gone.

- Found it so soothing. Provided coolness and decreased irritation.

- Can't possibly imagine how bad my radiation burns would have been without InterDry. Anytime it was off the pain was unbearable. As soon as I replaced it, pain and itch were instantly relieved.

- The cloth has provided so much comfort to me during the healing process.

- I went from crying to being comfortable with the burns.

- Thank you very much for providing this awesome medical dressing. I have really appreciated the wonderful service. Again, thank you for your time and consideration.

- Without this dressing I would not have been able to complete my treatment.

- I went from crying to being comfortable with the burns. The InterDry stopped the skin from blistering and gave me relief from the burning sensation all the time.

- I am so pleased, lucky to have been a participant in this project/ study. The cloth has provided so much comfort to me during the healing process. I truly hope it can be made available to all radiation patients in the very near future.

- The day I got the dressing I was itchy, prickly and burning. On the way home, I noticed I wasn't bothered by the symptoms anymore. The annoyance was gone.

Challenges

- Unable to keep dressing in place to under arm. Dressing worked well to the chest and where the dressing stayed in place.

- Found it so soothing. But need to figure out a better way to keep it in place.

\section{DISCUSSION}

This project was undertaken to evaluate the effects of InterDry Ag dressing for breast cancer patients receiving radiation treatment. Tanay (2013) reported that most women face negative emotional distress such as tension, embarrassment, isolation, fear and uncertainty due to physical side effects associated with radiation treatment. Clearly, there is a need to build trusting relationships from the time of diagnosis and throughout treatment to help patients ask his/her healthcare professional for medical guidance and emotional support (Bonito, 2013). Participants in this project shared their intimate experiences, including challenges of being diagnosed with $\mathrm{BC}$ and going through radiation treatment. A trusting relationship contributes to significant decrease in the level of distress, especially when initiating radiation treatments (Bonito, 2013).
Banning (2013) illustrates that feelings of powerlessness arise from the unequal health professional-client relationship that the general public perceives at the time of cancer diagnosis and treatment; thus being supported and empowered is important for patients to feel at ease.

Disruption of the integrity of skin due to radiation treatment is a common experience, resulting in wound repair process that is a well-orchestrated and complex phenomenon (Kramer, 2010). A randomized controlled study (Laffin et al., 2015) confirms that up to $41 \%$ of patients during and immediately following completion of radiation treatment for breast cancer in the tropics may develop moist desquamation of the skin. This requires utilization of various cancer care management strategies such as diverse dressings with analgesia. Patterson et al. (2012) confirm it is possible that women of different ethnicities perceive their skin reactions differently rather than there being any quantifiable differences in the degree or locality of erythema or desquamation.

Results of an in-depth analysis indicated that skin changes caused by breast cancer radiotherapy negatively affects numerous dimensions of women's quality of life, including physical well-being, functional well-being, and treatment satisfaction (Schnur, Ouellette, Dilorenzo, Green, \& Montgomery, 2011). Women in the current evaluation project expressed feelings of being safe, which gave them a sense of control over the emotional and psychosocial impacts of dealing with skin breakdowns due to the BC diagnosis and treatment. Weekly assessments by the clinic nurse offered a therapeutic sense of support and control for patients' inner emotional self and the physical side effects of treatments. Patients in the current project reported satisfaction from the weekly assessments that aided in fostering a trusting relationship between the nurse and patient. The telephone questionnaire seemed to play a large part in developing trust and further engaging patients to self-manage in their own care. A recent study conducted by Bonito (2013) discusses that cancer patients reported greater satisfaction with their healthcare professionals and improved quality of life when healthcare professionals discussed the emotional impact of cancer. They felt it gave them permission to share their experiences. Noble-Adams (1999) also reports that a thorough holistic skin assessment conducted by the nurse ensures optimal skin care during post radiation treatments. Participants expressed an appreciation of care and their ability in openly sharing their emotional well-being.

\section{LIMITATIONS}

Limitations of this evaluative project include the small sample size and a lack of participant diversity. A more diverse group of participants from other ethnicities who spoke other than English may have helped to identify gaps in the care provided for patients with radiation dermatitis. Patients of other ethnicities may also have indicated different self-care management practices. Future evaluative projects should be conducted with diverse groups to understand what type of educational material needs to be directed at non-English speaking women. Inclusion of family members who may be potential care givers would have been a critical improvement in the project design. 
This project could lend itself to a randomized trial with the use of a control group and validated tools. Data collection tools used in this project were developed from clinical observations but were not validated. Collection of further demographic data would also yield valuable data in terms of skin toxicity and the use of InterDry Ag dressing. A design conducted over a longer term would have also yielded key components of self-care management practices and feelings of self-efficacy.

\section{IMPLICATIONS FOR PRACTICE}

The InterDry Ag dressing has demonstrated its ability to decrease discomfort women experienced while receiving radiation treatment of the breast. A larger study using a randomized control design could help to further delineate the benefits of InterDry Ag dressing and its ability to maintain skin integrity in comparison to current standard management that heals radiation dermatitis. Within this small group of participants, InterDry Ag dressing appeared to be cost effective since majority of participants did not need a prescription for flamazine. Providing comfort for patients' physical and emotional needs during this difficult time is critical in maintaining a state of well-being. Teaching self-management skills for this group

\section{REFERENCES}

Banning, M.G. \& Gumley, V. (2013). Case studies of patient interactions, care provision and the impact of emotion: A qualitative study. Journal of Cancer Education, 28, 656-661.

Bonito, A.H., Horowitz, N., McCorkle, R., \& Chagpar, A.B. (2013). Do healthcare professionals discuss the emotional impact of cancer with patients? Psycho-Oncology, 22, 2046-2050.

British Columbia Cancer Agency (2013). British Columbia Symptom Management Guidelines. Retrieved from http://www.bccancer. bc.ca/health-professionals/clinical-resources/nursing/ symptom-management

Budischewski, K.F. (2008). Quality of life of breast cancer patients in the course of adjuvant radiotherapy. Support Care Cancer, 16, 299-304.

Canadian Cancer Society (2016). (n.d.). Breast cancer statistics. Retrieved from http://www.cancer.ca/en/cancer-information/ cancer-type $/$ breast/statistics $/$ ? region=bc

Gosselin, T.K., Schneider, S.M., Plambeck, M.A., \& Rowe, K. (2010). A prospective randomized, placebo-controlled skin care study in women diagnosed with breast cancer undergoing radiation therapy. Oncol Nurs Forum, 37(5), 619-626.

Gotz, C., \& Singh-Carlson, S. (2014). Mixed methods approach to program evaluation: Measuring impact of a cancer support program. Journal of Women's Health, Issues Q C Care, 3(3), 1-4. http://dx.doi.org/10.4172/2325-9795.1000151

Kennedy-Evans, K.L., Smith, D., Viggiano, B., Henn, T. (2007). Multi-site feasibility study using a new textile with silver for management of skin conditions located in skin folds. Journal of Wound, Ostomy a Continence Nursing, 34(3S), S68.

Kramer, A., Hubner, N.O., Assadian, O., Mulder, G. (2010). Polihexanide-perspectives on clinical wound antisepsis. Skin Pharmacology and Physiology, 23(Suppl 1), 1-3.

Kumar, S., Juresic, E., Barton, M., \& Shafiq, J. (2010). Management of skin toxicity during radiation therapy: A review of the evidence. Journal of Medical Imaging and Radiation Oncology, 54(3), 264-79. of patients could prove effective and contribute to a sense of empowerment. The InterDry Ag dressing enabled many participants to perform activities of daily living without the relentless pain and discomfort of a radiation skin reaction.

\section{CONCLUSION}

The use of InterDry Ag dressing for radiation skin reactions to the breast has shown to be beneficial in terms of providing comfort to this small cohort of patients. It is of utmost importance to maintain an individual's ability to conduct practical daily activities after receiving a $\mathrm{BC}$ diagnosis and the subsequent treatments. Most participants voiced their concerns and fears through a trusting relationship with the RON, which, in turn, provided comfort and relieved anxiety. This patient-centred approach allowed BC patients to experience personalized cancer care while journeying through the diagnosis and its related treatments.

\section{CONFLICT OF INTEREST}

The authors have no conflicts of interest to declare related to the products used for this project.

Laffin, N.S., Smyth, W., Heyer, E., Fasugba, O., Abernethy, G., \& Gardner, A. (2015). Effectiveness and acceptability of a moisturizing cream and a barrier cream during radiation therapy for breast cancer in the tropics. Cancer Nursing, 38(3), 205-2014.

NCI CTCAE (version 4.03). Dermatitis radiation (2013). Retrieved from, H:|everyone $\backslash$ nursing $\backslash$ references and guidelines $\backslash$ Symptom Management Guidelines \radiation dermatitis

Noble-Adams, R. (1999). Radiation-induced reactions. 1: An examination of the phenomenon. British Journal of Nursing, 8(17), 1134-40.

Porock, D., \& Kristjanson, L. (1999). Skin reactions during radiotherapy for breast cancer: The use and impact of topical agents and dressings, European Journal of Cancer Care, 8, 143-153.

Paterson, D., Poonam, P., Bennett, N.C, Peszynski, R., Beekhuizen, M.J.V., Jasperse, M.L., \& Herst, M. (2012). Randomized intrapatient controlled trial of Mepilex Lite Dressings versus Aqueous Cream in managing radiation-induced skin reactions postmastectomy. Cancer Science Q Therapy, 4(11), 347-356.

Schnur, J.O., Ouellette, S.C., Dilorenzo, T.A., Green, S., \& Montgomery, G.H. (2011). A qualitative analysis of acute skin toxicity among breast cancer radiotherapy patients. PsychoOncology, 20, 260-268.

Sibbald, G.R., Kelley, J., Kennedy-Evans, Labrecque, C., \& Waters, N. (2013). A practical approach to the prevention and management of intertrigo, or moisture-associated skin damage, due to perspiration: Expert consensus on best practice. Wound Care Canada, 11(2).

Smith, L.S., Singh-Carlson, S., Downie, L., Payeur, N., \& Wai, E. (2011). Survivors of breast cancer: Patient perspectives on survivorship care planning. Journal of Cancer Survivorship, 5, 337-44.

Stemler, S. (2001). An overview of content analysis. Practical Assess Res Evaluation, 7(17). Retrieved from http://pareonline.net/getvn. asp? $=7 \& n=17$

Tanay, M.W., Wiseman, T., Roberts, \& Ream, E. (2013). A time to weep and a time to laugh: Humour in the nurse-patient relationship in an adult cancer setting. Support Care Center, 22, 1295-1301. 


\section{APPENDIX A}

Skin Assessment-To be completed by RN

Indicate area of erythema or irritation.

\begin{tabular}{|l|l|l|l|l|}
\hline Physical Assessment & None & Mild & Moderate & Severe \\
\hline Erythema & & & & \\
\hline Pain (skin or muscle) & & & & \\
\hline Itchiness & & & & \\
\hline
\end{tabular}

Indicate area of erythema or irritation.

\begin{tabular}{|l|l|l|l|l|}
\hline Physical Assessment & None & Mild & Moderate & Severe \\
\hline Erythema & & & & \\
\hline Pain (skin or muscle) & & & & \\
\hline Itchiness & & & & \\
\hline
\end{tabular}

Indicate area of erythema or irritation.

\begin{tabular}{|l|l|l|l|l|}
\hline Physical Assessment & None & Mild & Moderate & Severe \\
\hline Erythema & & & & \\
\hline Pain (skin or muscle) & & & & \\
\hline Itchiness & & & & \\
\hline
\end{tabular}

1. Are you presently using the InterDry AG? (If no, proceed to questions $10 \& 11$ )

2. Do you find the dressing easy to use?

3. Does the dressing relieve any discomfort you are experiencing?

4. Have you changed the dressing more than every five days?

5. If you have pain has the dressing decreased your pain?

6. Is the dressing comfortable?

7. Do you feel the dressing has helped your skin to heal?

8. Does your skin feel better with the dressing on or off?

9. Why did you stop using the dressing? (only applicable if not using the dressing).

10. Are you using any other dressings or creams?

11. Do you have any questions or concerns you would like to discuss? 


\section{APPENDIX B}

Telephone Interview (conducted by nurse)

Follow-up assessment of InterDry AG

Hello may I please speak to ?

Hello my name is and I am calling from the BCCA. I am calling in regards to your one week follow-up with the use of InterDry AG. Do you have a few minutes to discuss how you have been doing over the past two weeks?

To Begin.... How are you?

1. Are you presently using the InterDry AG? (If no, proceed to questions \#10 \& 11)

2. Do you find the dressing easy to use?

3. Has the redness of your skin worsened or improved since your last treatment date?

4. Has your skin opened since your last treatment? ie: Are there any wet patches?

5. Have you changed the dressing more than every five days?

6. Do you have any pain? If so would you describe the pain as skin or muscle pain?

7. Is the dressing comfortable?

8. Do you feel the dressing has helped your skin to heal?

9. Does your skin feel better?

10. Why did you stop using the dressing? (only applicable if not using the dressing).

11. Are you using any other dressings or creams?

Do you have any questions or concerns you would like to discuss?

Thank you for your time and valuable feedback. We will see you in another week on @ If you have any questions or concerns please call the Nursing telephone line @ 604-930-4053. Have a nice day. Goodbye. 


\section{APPENDIX C}

Patient Assessment of InterDry AG

\begin{tabular}{|c|c|c|c|c|c|c|}
\hline $\begin{array}{l}\text { How strongly do you agree with } \\
\text { this statement? }\end{array}$ & Strongly Agree & Agree & Undecided & Disagree & Strongly Disagree & N/A \\
\hline \multicolumn{7}{|l|}{ 1. InterDry AG is easy to use. } \\
\hline \multicolumn{7}{|l|}{$\begin{array}{l}\text { 2. InterDry AG provides relief of } \\
\text { pain. }\end{array}$} \\
\hline \multicolumn{7}{|l|}{ 4. The Dressing is comfortable. } \\
\hline \multicolumn{7}{|l|}{$\begin{array}{l}\text { 5. My skin feels better with the } \\
\text { dressing on. }\end{array}$} \\
\hline \multicolumn{7}{|l|}{$\begin{array}{l}\text { 8. I have used the dressing for five } \\
\text { days without having to replace it. }\end{array}$} \\
\hline \multicolumn{7}{|l|}{$\begin{array}{l}\text { 9. I feel more comfortable with the } \\
\text { dressing in place than without the } \\
\text { dressing. }\end{array}$} \\
\hline $\begin{array}{l}\text { 10. I would recommend this dress- } \\
\text { ing to others. }\end{array}$ & & & & & & \\
\hline
\end{tabular}

11. Any further comments regarding the use of InterDry AG? 